\title{
The Potential of Drone Technology in Pandemics
}

\author{
David R. Green, Alex R. Karachok and Billy J. Gregory
}

In recent years, drones or Unmanned Airborne Vehicles (UAVs) have become associated with aerial data and image acquisition for many environmental applications. Unexpectedly, the COVID-19 pandemic in 2020 has led to new opportunities for drones in many new application areas, with and without the use of remote sensing imaging technology. Many unique applications have already been proposed within a short period of time, some building on existing pre-COVID-19 demonstrations and ideas, to exploring new possibilities for use. Whilst drone technology is well-established, there are still factors requiring further investigation to fully exploit drones. These include: allocation, management and control of drone air space, operation Beyond Visual Line of Sight (BVLOS), the role of existing and new telecommunication networks, safe operation in built-up areas, and societal approval and acceptance. This chapter presents an overview of current drone technology and future developments and the exploration of some existing and proposed applications. The chapter will examine the issues facing successful mainstream implementation of the use of drones for these applications, and the problems that need to be overcome to allow this technology to mature and become mainstream.

\subsection{Introduction}

Drones or UAVs first became popular about six years ago when Parrot, 3DR, and DJI began to market their off-the-shelf platforms to the public. Categorised mainly as 'toys' at first these low-cost multi-rotor aircraft rapidly became very popular for recreational flying. Their widespread appeal soon grew as they became easier to fly and could carry small cameras. The addition of GoPro Hero cameras and DJI Zenmuse gimbals helped to facilitate and reveal the potential of these small airborne platforms and sensors for aerial photography and videography.

Although the future potential of drones for applications outside remote sensing and the derived photogrammetric and visualisation products we have become so familiar with were already under consideration prior to COVID-19, the widespread impact of the pandemic has triggered a rapid escalation in interest in the role of drone technology. Already the COVID-19 pandemic has provided a new opportunity to explore the potential role of drones in other application areas, both with and without the use of remote sensing technology. Many unique applications have already been proposed within a short period of time, some capitalising on existing demonstrations and ideas, to those exploring new possibilities.

Two streams of application have been considered: (1) delivering essential goods and services, and (2) battling the spread of coronavirus. Some examples already under development and testing include: the delivery of parcels, medical supplies, and information e.g. broadcasts; personal, health and environmental monitoring, enforcing social distancing, mapping, and spraying to disinfect. 
Whilst drone technology is already well-established, there are still many factors that currently require further investigation to allow this proposed potential to be fully exploited. These include the allocation, management and control of drone air space, operation Beyond the Visual Line of Sight (BVLOS), the use of new telecommunications networks, safe operation considerations in built-up areas, as well as social acceptance. With this context in mind some of the existing and proposed applications that are either already under development or being developed will be explored. This will include an overview of current drone technology and future developments. The chapter will then look at the issues facing successful implementation of the use of drones for these applications, and the problems that need to be overcome to allow this technology to become mainstream in the future.

\subsection{Developments in Drone Technology}

Today there are a wide range of UAV and drone platforms which include multi-rotors (quadcopter (4), hexacopter (6), and octacopter (8)), fixed wing, and Vertical Takeoff and Landing (VTOLs). In addition, some small-scale helicopters have also been used for remote sensing. High quality gimbals now support a wide range of DSLR cameras of varying different sizes, and additional camera-based sensors, such as NIR and NDVI cameras. Larger cameras and sensors can be carried by bigger drones with the required lift and battery capacity.

There are now many more options for sensors that can be mounted on small aerial platforms. Whilst RGB cameras are generally still in widespread common use, the demand for other sensors, both non-imaging and imaging, and low-cost, higher resolution systems has seen many other types of cameras/sensors become available. These include modified GoPro cameras and similar types of miniaturised cameras that can be filtered to sense the Near Infrared (NIR) and other portions of the Electromagnetic Spectrum (EMS). Examples include the range marketed by MapIR [1] and IR-Pro in the USA. Alongside these have been developments in small multi-spectral sensors specifically aimed at agricultural, horticultural crop and forest canopy monitoring. The Parrot Sequoia 5-band multi-spectral camera is one such example [2]. A larger version of this sensor, is the MicaSense RedEdge [3]. These provide the opportunity to acquire five (5) bands of imagery, and also to generate NDVI images. Other companies such as Sentera (www.sentera.com) also provide NDVI cameras based on different spectral wavelengths.

More advanced sensors are now also available to take greater advantage of the information content of the electromagnetic spectrum including thermal, hyperspectral, and Lidar instruments. Many drone manufacturers offer thermal camera-ready platforms with either their own thermal cameras e.g. Yuneec [4] and DJI [5] or ones made by a thermal camera manufacturer e.g. FLIR. Hyperspectral cameras are available for drones from a number of different manufacturers e.g. Headwall [6]. These can be useful in many applications because of the number of wavelengths that can be sensed (e.g. up to 255 channels) greatly increasing the dimensionality of the spectral dataset and resulting in the potential acquisition of more information of interest. These are expensive, often need specialist aerial platforms, and require more training to use and to extract the information. Another common sensor is Lidar (both terrestrial and bathymetric versions) which can be mounted on a number of specialist UAV platforms e.g. Riegl [7]. Terrestrial lidar has the potential to see through a vegetation canopy. Bathymetric lidar sensors can be mounted on small aerial platforms and have the capability to generate depth information in water $20-40 \mathrm{~m}$ deep providing the water is clear and not too turbulent e.g. Riegl's Bathycopter [8]. These also have the capability to generate $3 \mathrm{D}$ information of underwater surfaces.

Radar systems have also been integrated into large UAV systems for navigation and image acquisition. More recently, studies have revealed success with the development of small, low-cost, high-resolution radar systems specifically designed for operation on small unmanned aerial vehicles [9-11].

In a very short period of time, drone technology has evolved from being quite basic to 
very advanced and increasingly customised for specific environmental applications. Several key developments in the technology have facilitated this transformation.

One of the major constraints of early platforms was the available battery technology. Early batteries provided a limited power source often only giving 6-8 minutes of flight time. This was not a major drawback for short recreational flights, but when including considerations such as operation in colder air, wind strength, and take-off, return to home, and landing requirements, the actual flight duration time was reduced quite considerably. Within a few years, however, these basic batteries have evolved into smart batteries that not only provide longer flight times e.g. 20-25 minutes, but also supply information to the controller and operator about battery status and remaining flight time. Within a few years these batteries have also become more compact and the average flight time has now increased to around 30 minutes. Considerable effort is now being put into improving the quality and battery life, as well as the development of new types of batteries [12], especially for multi-rotors. Fixed-wing aircraft by contrast typically already have longer flight times, ranging from 45 to 55 minutes, as they are lighter and offer less resistance in flight, making them more suitable for larger area coverage.

Early UAVs were usually flown manually by the pilot, often without an First Person View (FPV) device, and stereo-imagery was acquired through skilled flying by the drone pilot where overlapping flight-lines were visually assessed to provide the required stereo-imagery for generating photo-mosaics and 3D models. Today sophisticated phone and tablet Apps for both Android and iPhone operating systems, either provided by the drone companies (e.g. DJI Go4) or third party companies (e.g. Litchi [13], and Pix4D [14]), allow for varying levels of autonomous flight to be planned and executed. The phone and tablet Apps now available allow for either very basic simple autonomous flight (Litchi) or more sophisticated grid pattern flightpaths (Pix4D) to be conducted that allow the pilot to customise the overflight pattern e.g. straight line or grid, the camera tilt, speed, and the flying height of the aircraft amongst other things. Over time, the data/image capture task has now become a one-button 'push' for take-off and one for landing. The technology has therefore made it much easier to conduct repeat flights of the same area to gather multi-temporal imagery of a site. Nearly all modern drones now have autonomous flight capability, and this in turn allows them to be used for more advanced aerial survey tasks.

Additionally, some drones (e.g. the DJI Mavic Air 2) now come equipped with the new AirSense technology utilising ADS-B aviation technology for receiving signals from nearby airplanes and helicopters and displaying their locations on the on-screen map on the DJI Fly App [15].

Not surprisingly the growing number of different UAVs and the range of applications is already beginning to raise concerns about the availability and safe use of air space particularly where other industry areas will also be affected by competition for the existing air space. This will ultimately require legislation. UAV communication with the ground control system (GCS), for example, requires radio frequencies with sufficient band width. As recently as 2008, the International Telecommunication Union (ITU) had not allocated bandwidth to UAVs meaning that they have to use different radio frequencies in every country [16], something that needs to be taken into account by international operators and manufacturers.

Several drone capture methods have also been developed either to physically capture drones in a net or through 'drone snatching' using another drone in situations where illegal flights are being conducted or in emergency situations $[17,18]$. All weather drone operation is now being developed and delivery drones are already able to safely operate in heavy rain and high winds [19].

\subsection{The Impact of COVID-19}

Prior to the COVID-19 pandemic, although drone technology was already well-established with many professional applications worldwide there were already a number of new ideas emerging for their use in a wider range of imaging and non-imaging applications.

At the time, drones were also increasingly coming under scrutiny because of the increasing occurrence of operational incidents. Though not all were substantiated, some accidents nevertheless 
began to have a negative impact on the perception of this technology leading to alienation of the public from supporting the continued growth of this industry. In part this was a direct result of the rapid growth in uncontrolled use of both commercial and recreational drones, with a lack of pilot training and enforcement of regulations. However, responses to concerns about privacy and safety also had many positive developments leading to improved technology, education and awareness raising, training, best practice guidelines and tighter regulations that have since helped to allay some public concern.

The COVID-19 pandemic, however, rather suddenly and unexpectedly triggered growing recognition and a new level of acceptance of drone technology, driven not so much by the increase in commercial uses prior to the outbreak of COVID-19, but by the timely emergence of new ideas that have highlighted drone technology as an important way to help deal with and, possibly even overcome, the constraints and health emergencies imposed on society by the virus in early 2020 . In addition, the collection of higher resolution data that can be processed and integrated with other information for planning and decision-making.

Two new streams of application have been pursued: (1) delivering essential goods and services, and (2) battling the spread of coronavirus. Some examples already under development and being tested include: the delivery of parcels, food, medical supplies, and information e.g. broadcasts; personal, health and environmental monitoring, enforcing social distancing, mapping, and spraying to disinfect contaminated areas. These are explored below.

\subsubsection{Delivering Essential Goods and Services}

Prior to the arrival of COVID-19, numerous articles and press releases revealed a growing commercial interest in the use of so-called 'delivery drones' for the delivery of parcels, supplies, food. and drink. It has been predicted that by 2030 the drone package delivery market will be worth £21bn [20]. Similar forecasts have been made by others about the impact of this technology e.g. by 2026, more than a million drones could be carrying out retail deliveries, up from 20,000 today, according to new analysis from Gartner [21].

Amazon and a number of other companies were originally at the forefront of promoting these ideas, and successful demonstrations had already been carried out as proof of concept. Prime-Air, for example, was first trialled by Amazon in 2016 to demonstrate parcel delivery within a 30 minute timeframe [22]. Prime-Air development centres were set up in the United States, the United Kingdom, Austria, France and Israel. Research studies showed that drone platforms already available - albeit larger than some of the more familiar recreational drones - could easily be adapted and modified to carry small boxes and containers. Coupled with new autonomous flight capability it has since been shown possible to programme a drone to carry a cup of coffee or a meal from point A to point B. Larger platforms were also shown to be capable of carrying larger payloads such as parcels. DHL also launched drone operation tests in urban areas in China to test the so-called 'last-mile' delivery challenges being considered. Compared to road transport, the benefits of autonomous drone services were found to be significant and included a reduction in delivery times, and provided cost savings, reduced energy consumption, and a lower carbon footprint [21]. In addition, such drones were able to provide faster delivery for customers with the added benefit of giving consumers more control over the time and location of a delivery [19].

The capability of larger drones to carry boxes also opened up the possibility of carrying supplies to remote and inaccessible locations (e.g. in the event of an emergency or for delivering medical supplies) where timing is often important. Several trials have been undertaken and revealed the potential to expand drone-based deliveries to more applications. For example, in Canada, they have been delivering supplies, including medical supplies, to native communities in places where there are a lot of small, remote reserves far from resource hubs [23].

However, despite the potential there are many drone-related issues that require early solutions to allow for the practical realisation of these ideas in the future e.g. the establishment of an air traffic management system. In addition, there have been some concerns that drones maybe the target of 'hackers' who may be able to take control of a drone and steal the goods being carried.

Meantime, on a more positive note these ideas and developments have also begun to find favour 
with roles that might be useful to help deal with the actual and perceived impact of the COVID-19 pandemic.

The collection of high-resolution spatio-temporal data can provide the means to generate real-time information e.g. images, maps, and surveys at the community level to assist in planning and decision-making, as well as for use in an emergency response both during and after an event.

A recent report in 2020 highlights the opinion that the pandemic will be 'a catalyst for accelerating the viability and acceptance of commercial drone deliveries' [24]. 53 per cent of companies who took part in a recent survey believe commercial drone deliveries will be commonplace by 2023, helped by the public and private sector actively seeking to develop safe ways to guarantee services whilst containing the spread of any viruses [24].

Drone delivery has the potential to provide people with low-cost and near instant access to vital medical supplies, regardless of whether they live in a city or a rural community. Benefits arising will be in helping to manage chronic health conditions, enabling more home-based acute care, and reducing the number of hospitalisations and trips to accident and emergency rooms [19].

Drones have been used to deliver medical supplies in the Dominican Republic [25].

\subsubsection{Battling the Spread of Coronavirus}

Aside from using drones being used to carry goods, many drones are also equipped with cameras, microphones and speakers and so can be used for monitoring and to both gather and deliver information. In this respect they will utilise existing functionality that has not necessarily been used in this way before.

An area of application where there is now immense potential for drone use lies with personal, health and environmental monitoring, enforcing social distancing, mapping, and spraying to disinfect. These are all ways where drones may help to reduce the spread of the corona virus. As drones typically carry cameras - whether RGB colour or thermal - they can be used both indoors and outdoors as a remote monitoring platform.

The growing use of custom-built drone platforms carrying thermal cameras to monitor heat loss from buildings, for firefighting applications, and is also being proposed (and has already been used) to help monitor the health of people where temperature can be used as an indicator of possible infection from the corona virus. The benefit of having an airborne platform is obviously that it is in the air, far from contacting humans and can still return accurate results [26].

In Canada, Draganfly is adapting scanning technology for drones to combine thermal imaging systems and a computer program with learning algorithms to monitor temperature, heart and respiratory rate at a distance to aid in the detection of someone sneezing or coughing, and can be used for screening [27].

This is basically an extension of the more traditional use of cameras for remote sensing of surfaces that provides imagery for automated or manual interpretation. Some commercial drones (e.g. the DJI Mavic Enterprise) also carry loudspeakers that can be used to communicate information to individuals on the ground with one application being the provision of messages to help enforce social distancing in public spaces. The use of drones to communicate information and messages can also be used in many other environmental monitoring or situation scenarios. Having a remote video platform that can both record and broadcast live is a much better alternative than putting someone at risk to film by hand. You also get a more holistic picture, and can easily document dangerous gatherings from a distance without having to get close to people who are violating the pandemic regulations.

The capability to carry a larger payload also allows drones to carry other materials and equipment. One example, which is an extension of the application of drones for spraying crops, is the use of drones to carry disinfectant and spray equipment to disinfect environmental locations or internal rooms. Cities have also been using Drones such as DJI's agricultural drones to spray disinfectant on an area, which is a lot more cost-efficient than employing a team of workers using expensive and equipment to spread disinfectant by hand on the ground [28, 29]. In Scotland, a UK company Droneports has recently demonstrated the capability of drones being used to support the NHS by delivering COVID-19 test kits and medical supplies to an island off the coast of the 
Scottish mainland. The demonstration showed how supplies could be delivered more quickly to the island than the normal route used by road and ferry [30].

Similar trials, which are now being fast-tracked in light of the pandemic, have also been reported in other parts of the UK [31].

During the COVID-19 crisis, drones have already been used to deliver medication and test samples in other remote locations in Ghana, Rwanda, and Chile. In Charlotte, North Caroline, USA, drones have been used to deliver personal protective equipment and supplies to frontline medical teams. This initiative was part of the North Carolina Department of Transportation's (NCDOT's) Unmanned Aircraft System Integration Pilot Program (IPP).

Unmanned aerial vehicles have also been used in several cities around the world to monitor compliance with virus-related safety measures as well as to spray disinfectant e.g. in India and China [21]. China was in fact one of the first countries to use drones in response to COVID-19 and as early as February 2020, Antwork - part of the Japanese group Terra Drone - used a drone to transport test samples and medical supplies from a local hospital in Zhejiang province to a nearby disease control centre. In 2019, Antwork were the first urban drone delivery company to obtain a license from the Civil Aviation Administration of China (CAAC). Some of the benefits arising from this demonstration were that the transport time was halved, and this in turn was found to relieve stress on medical staff.

In April 2020, Manna Aero were approved by Ireland's aviation authority to deliver medication and critical supplies to roughly a dozen households under confinement. In Switzerland, Matternet a US company - had already been cleared to carry out autonomous, beyond-the-line-of-sight flights for the transport of blood samples between hospitals in the city of Lugarno. Another US-based drone operator, Zipline supported Ghanaian authorities by providing a 'contactless drone delivery' service to collect Coronavirus test samples from 1000 rural health facilities and to deliver them to laboratories in Accra and Kumasi [32]. These are just a few of the practical demonstrations that have already been developed to highlight the potential of autonomous aerial technology to assist in emergencies.

Other mentioned uses of drones include the delivery of non-medical products such as groceries and other food supplies to people and communities, and to extend the already well-tried data acquisition role that they have been demonstrating in the field of mapping, which sees drones providing real time imagery for emergency response and near real time mapping. The extension of the mapping role to areas that have not been mapped at certain scales also has potential in the context of COVID-19. 


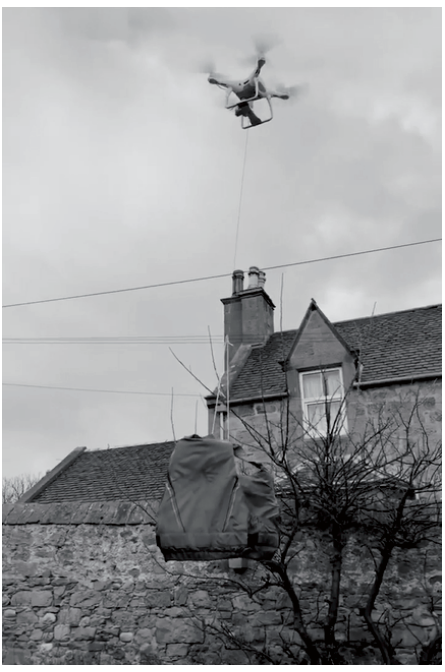

(a)

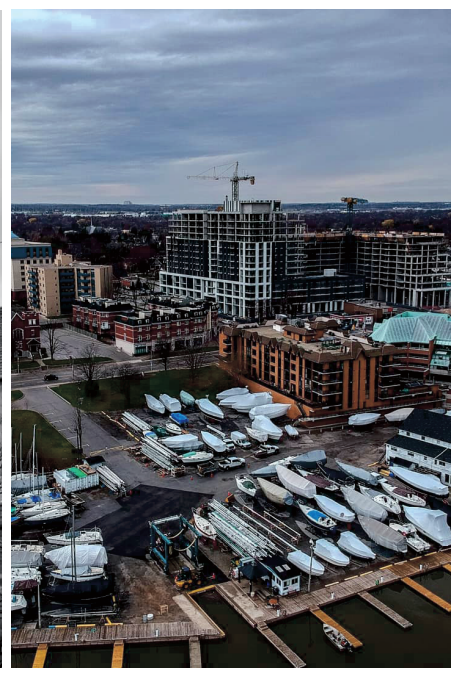

(b)

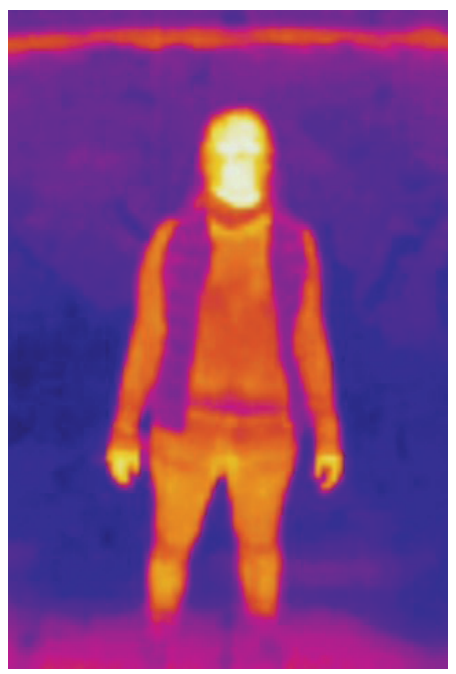

(c)

FIGURE 5.1

a) A Quadcopter Drone Lifting a Bag (Billy J. Gregory - DroneLite); b) Monitoring urban areas during lockdown (Alex R. Karachok - UCEMM); c) A thermal camera image (Billy J. Gregory DroneLite)

\subsection{Summary and Conclusions}

Drones began as recreational toys, a modern-day, updated radio-controlled model aircraft but with the added benefits provided by the evolution of the technology. Recognition of their potential for many commercial applications soon raised the public profile of drones and their many uses. However, various incidents with drones involved in injuries and near misses with civilian aircraft have somewhat dented the positive perception of drone technology with the public threatening to derail future growth of the market.

With the sudden and very significant impact of COVID-19 worldwide and the need for monitoring, mapping, and social distancing to minimise contact between people and to help reduce the spread of infections, robotic technologies were soon recognised as one way by which many aspects of the pandemic could be monitored and assisted. As a direct result of this, drone technology is gradually beginning to find growing support although it is still true to say that a number of issues are still a cause for concern.

The practical experience gained so far during the COVID-19 pandemic has been both stimulating and has undoubtedly paved the way for developments needed to make better use of drone applications in the future. Furthermore, lessons learned during the crisis and from examining the use-cases that have proved to be of societal benefit during the corona virus crisis will very likely encourage updates to drone regulations that go beyond the use of drones in times of crisis enabling the wider use of drone technologies in the future.

The ability to enhance the collection of up-to-date high resolution data over both space and time using drones (aerial, terrestrial, or waterborne) will provide a basis for more detailed information that can help emergency response and planning teams in the context of building community resilience frameworks in the future.

However, significant developments in the technology are still needed to overcome some of the public concerns and to allow drone applications to reach their full potential. This includes major 
advances in existing telecommunications, air traffic management systems, and regulations amongst a number of other things.

Being at a stage when we are now already able to successfully utilise and adapt current drone technology to conduct numerous activities safely and remotely has been very timely in light of the COVID-19 pandemic.

The progress made during this pandemic will undoubtedly help society to function more safely and more effectively during times of crisis in the future whereby drone technology will be able to provide the data and information needed to assist in an emergency response, whilst also being able to assist communities to build future resilience.

\section{References}

[1] MAPIR. Mapir cameras, 2020. URL https://www.mapir.camera/. Accessed on 27 September 2020.

[2] Parrot. Parrot drones sequoia camera, 2020. URL https://www.parrot.com/uk/shop/accessories-spare-parts/ other-drones/sequoia. Accessed on 27 September 2020.

[3] MicaSense. Micasense rededge sensor, 2020. URL https://micasense.com/rededge-mx/. Accessed on 27 September 2020.

[4] Yuneec. Yuneec drones, 2020. URL https://www.yuneec.com. Accessed on 27 September 2020.

[5] DJI. Dji drones, 2020. URL https://www.dji.com. Accessed on 27 September 2020.

[6] Headwall Photonics. Headwall sensor drone integration, 2020. URL https://www.headwallphotonics.com/uavintegration. Accessed on 27 September 2020.

[7] Riegl. Riegl laser management systems, 2020. URL http://www.riegl.com/. Accessed on 27 September 2020.

[8] Riegl. Riegl bathycopter, 2020. URL http://www.riegl.com/products/unmanned-scanning/bathycopter/. Accessed on 27 September 2020.

[9] Li, CJ and Ling, H. Synthetic aperture radar imaging using a small consumer drone. In International Symposium on Antennas and Propagation \& USNC/URSI National Radio Science Meeting, pages 3-9. IEEE, 2015.

[10] Scannapieco, AF, Renga, A, Fasano, G, and Moccia, A. Ultralight Radar for Small and Micro-UAV Navigation. In International Archives of the Photogrammetry, Remote Sensing and Spatial Information Sciences, International Conference on Unmanned Aerial Vehicles in Geomatics, volume XLII-2/W6, pages 333-338. IEEE, 2017.

[11] Ball, M. High-resolution sar imagery captured with small uas. unmanned systems technology, 2019. URL https://www. unmannedsystemstechnology.com/2019/02/high-resolution-sar-imagery-capturedwith-small-uas/. Accessed on 27 September 2020.

[12] Unifly. Breakthrough in new type of battery for drones, 2020. URL https://www.unifly.aero/news/ breakthrough-in-new-type-of-battery-for-drones. Accessed on 27 September 2020.

[13] Litchi. Litchi software, 2020. URL https://flylitchi.com/. Accessed on 27 September 2020.

[14] Pix4D. Pix4d software, 2020. URL https://www.pix4d.com/. Accessed on 27 September 2020.

[15] Abbott, J. Dji mavic air 2 review - the best drone around for beginners and hobbyists, 2020. URL https: //www.techradar.com/uk/reviews/dji-mavic-air-2-review. Accessed on 27 September 2020.

[16] Everaerts, J. The Use of Unmanned Aerial Vehicles (UAVs) for Remote Sensing and Mapping. Proceedings of The International Archives of the Photogrammetry, Remote Sensing and Spatial Information Sciences, XXXVII Part B1:1187-1191, 2008.

[17] Skitmore, A. Launch and Recovery System for Improved Fixed-Wing UAV Deployment in Complex Environments. Unpublished Masters Thesis. Department of Maritime and Mechanical Engineering. Liverpool John Moores University, 2018.

[18] Skitmore, A. Launch and Recovery System for Improved Fixed-Wing UAV Deployment in Complex Environments. Chapter 18. In, Green, DR, Gregory, BJ, and Karachok, AR, 2020 (Eds.). Unmanned Aerial Remote Sensing: UAS for Environmental Applications, CRC Press. 324p, 2020. 
[19] McKinsey \& Company. Debate: Will delivery uavs scales by 2030, 2020. URL https://www.mckinsey.com/ industries/capital-projects-and-infrastructure/our-insights/debate-will-delivery-uavs-scale-by-2030. Accessed on 27 September 2020.

[20] Ford, J. Skyports join 'beyond visual line of sight' uav trials, 2020. URL https://www.theengineer.co.uk/ skyports-bvlos-uav-caa-sandpit/. Accessed on 27 September 2020.

[21] Wray, S. Cities should prepare for an increase in delivery drones, 2020. URL https://cities-today.com/ cities-should-prepare-for-an-increase-in-delivery-drones/. Accessed on 27 September 2020.

[22] Amazon Prime Air. First prime air delivery, 2020. URL https://www.amazon.com/Amazon-Prime-Air/b?ie= UTF8\&node $=8037720011$. Accessed on 27 September 2020 .

[23] D'Sa, P. Drones will deliver covid-19 supplies to remote beausoleil first nation, 2020. URL https://www. google.com/amp/s/m.huffingtonpost.ca/amp/entry/drone-covid-first-nation_ca_5edea533c5b6fd8a1a2912a0/. Accessed on 27 September 2020.

[24] The Engineer. Covid-19 catalyst for commercial drone deliveries, 2020. URL https://www.theengineer.co. uk/commercial-drone-deliveries-protolabs-report/. Accessed on 27 September 2020.

[25] DJI. Dji - delivering the future of healthcare, 2020. URL https://youtu.be/TnXySwo8g7M. Accessed on 27 September 2020.

[26] Kiro7. Drones detecting body temperature being used in covid-19 response, $2020 . \quad$ URL https://www.kiro7.com/news/local/drones-detecting-body-temperature-being-used-covid-19-response/ CAGP3UM2IRCI7HMXPZMOL700XY/. Accessed on 27 September 2020.

[27] Burke, D. New reality: a revolution in infectious disease testing could be just overhead, 2020. URL https://www.cbc.ca/news/canada/nova-scotia/drones-covid-19-detection-computers-pandemictechnology-1.5534104. Accessed on 27 September 2020.

[28] Reagan, J. Eaglehawk deploys disnfectant drones to sanitize facilities, 2020. URL https://www.google.com/ amp/s/dronelife.com/2020/05/05/disinfectant-drones-eaglehawk/amp/. Accessed on 27 September 2020.

[29] Syracuse. Syracuse company develops coronavirus-killing drone for arenas and stadiums, 2020. URL https://www. syracuse. com/coronavirus/2020/05/syracuse-company-develops-coronavirus-killing-dronefor-arenas-and-stadiums.html. Accessed on 27 September 2020.

[30] Press Association 2020. Covid-19 test kit delivery drones receive funding boost, 2020. URL https ://www . eastlothiancourier . com/news/national-news/18573416. covid-19-test-kit-delivery-dronesreceive-funding-boost. Accessed on 27 September 2020.

[31] Mee, E. Drones will deliver medical supplies in trial, transport secretary says, 2020. URL https://news.sky.com/story/coronavirus-drones-will-deliver-medical-supplies-in-trial-transportsecretary-says-11978376. Accessed on 27 September 2020.

[32] International Transport Forum. Drones in the era of coronavirus, 2020. URL https://www.itf-oecd.org/ sites/default/files/drones-covid-19.pdf. Accessed on 27 September 2020 
$\Longrightarrow$ Taylor \& Francis Taylor \& Francis Group

http://taylorandfrancis.com 